\title{
The Osteogenic Effect of Local Delivery of Vancomycin and Tobramycin on Bone Marrow Stromal Cells
}

\author{
Lingjia Yu' \\ Qi Fei (DI \\ Jisheng Lin' \\ Yong Yang' \\ Yisheng $X u^{2}$ \\ 'Department of Orthopedics, Beijing \\ Friendship Hospital, Capital Medical \\ University, Beijing 100050, People's \\ Republic of China; ${ }^{2}$ Orthopedics \\ Department, Guangdong Provincial \\ Hospital of Traditional Chinese Medicine, \\ Guangzhou, Guangdong 510120, People's \\ Republic of China
}

This article was published in the following Dove Press journal: Infection and Drug Resistance

Purpose: Bone tissue infections are a difficult problem in orthopedic surgery. Topical application of vancomycin and tobramycin powder has been proved to significantly reduce infection rates. However, the osteogenic effect of the topical application of these two antibiotics is unclear. In this study, the osteogenic effect of local delivery antibiotics on bone regeneration was investigated in vitro.

Methods: Bone marrow stromal cells (BMSCs) were incubated in the presence of vancomycin $(14.28 \mu \mathrm{g} / \mathrm{mL})$, tobramycin $(28.57 \mu \mathrm{g} / \mathrm{mL})$, or vancomycin combined with tobramycin (vancomycin $14.28 \mu \mathrm{g} / \mathrm{mL}$ and tobramycin $28.57 \mu \mathrm{g} / \mathrm{mL}$ ). Cell viability, proliferation, and migration were analyzed. The alizarin red staining as well as the alkaline phosphatase staining was investigated. Then, the quantitative real-time (qRT)-PCR of osteogenic mRNA expression levels were also evaluated.

Results: The results showed that vancomycin combined with tobramycin has no adverse effect on the viability and proliferation of BMSCs. The topical application of vancomycin alone may interfere with the bone regenerative processes. However, the tobramycin can promote the osteogenic differentiation of BMSCs and also rescue the osteogenic potential of BMSCs inhibited by vancomycin both in vitro.

Conclusion: From this in vitro study, local application of vancomycin combined with tobramycin does not affect the osteogenic potential of BMSCs.

Keywords: vancomycin, tobramycin, osteogenesis, bone regeneration

\section{Introduction}

Orthopedic surgical infections could lead to delayed union or nonunion of fractures, delayed rehabilitation, increased re-operation rates, and increased morbidity and mortality. Prophylactic systemic intravenous antibiotics could reduce the surgical site infection rate 2 to 4 -fold. However, the local application of antibiotics after orthopedic surgery has been shown to reduce infection rates as much as 10 -fold. ${ }^{1}$ Furthermore, the local delivery of antibiotics can reduce antibiotic resistance and the undesirable effects related to systemic administration, such as ototoxic, nephrotoxicity. ${ }^{2}$ Thus, the local administration of antibiotic powder has become a prophylactic measure to help reduce bacterial infection in many orthopedic procedures. Many clinical studies have advocated that the use of vancomycin or tobramycin in orthopedic procedures is safe and could reduce the infection rate. ${ }^{3,6}$

Vancomycin is a widely used glycopeptide, which is indicated for the treatment of serious infection caused by methicillin-resistant staphylococcus aureus (MRSA). ${ }^{7}$
Correspondence: Yong Yang

Department of Orthopaedics, Beijin

Friendship Hospital, Capital Medical

University, Beijing 100050, People's

Yisheng $\mathrm{Xu}$

Orthopedics Department, Guangdong

Provincial Hospital of Traditional Chinese

Medicine, Guangzhou, Guangdong

5 I0I20, People's Republic of China

Email xuyishengdr@gzucm.edu.cn 
Tobramycin which has been proved to be an excellent drug for anti-infection belongs to the aminoglycoside family. It has a narrow spectrum and is active against Gram-negative bacteria infection, particularly against species of pseudomonas aeruginosa. ${ }^{8}$ Both two antibiotics have a low antigenicity and present a low allergy rate. Therefore, the combination of topically used vancomycin and tobramycin powder may provide a broad spectrum of antimicrobial activity including the majority of pathogens encountered in orthopedic surgery.

Several studies have shown that local administration of antibiotics may have cytotoxic effects. An in vitro study showed that intrawound vancomycin might interfere with the bone regeneration process and increase the risk of nonunion. ${ }^{9}$ Furthermore, an in vivo study demonstrated that local vancomycin treatment could reduce the heterotopic ossification formation in the rat model. ${ }^{10}$ Recently, Ishida et $\mathrm{al}^{3}$ demonstrated in a rat model of spinal fusion that intraoperative local application of vancomycin might detriment the fusion-mass formation, while tobramycin appeared to augment spinal fusion-mass formation. To best of our knowledge, no study has focused on the effect of topical delivery of vancomycin combined with tobramycin on bone regeneration in vitro.

This study aimed to investigate the osteogenic effects of vancomycin combined with tobramycin on viability, proliferation, migration, and osteogenic differentiation of Bone Marrow Stromal Cells (BMSCs) in vitro.

\section{Methods}

\section{BMSCs Culture}

Human BMSCs were purchased from Cyagen (USA) and cultured in human BMSCs basal media (Cyagen, USA) with $10 \%$ fetal bovine serum (Cyagen, USA), 1\% penicillin-streptomycin (Cyagen, USA), and glutamine (Cyagen, CA, USA). And osteogenic induction was conducted by BMSCs osteogenic differentiation media (Cyagen, USA) with 10\% fetal bovine serum (Cyagen, USA), glutamine (Cyagen, CA, USA), 1\% penicillin-streptomycin (Cyagen, USA), ascorbic acid (Cyagen, CA, USA), $\beta$-Glycerolphosphate disodium (Cyagen, USA), and dexamethasone (Cyagen, USA). All the cells were cultured at $37^{\circ} \mathrm{C}$ in saturated humidity containing $5 \% \mathrm{CO}_{2}$. The media were changed every two days. The BMSCs were digested using $0.25 \%$ trypsin (Solarbio, China), when they grew to a confluence of $70-80 \%$. To investigate the effect of topical antibiotics, BMSCs were cultured without antibiotics (Con group) or with antibiotics, which further subdivided into three subgroups: Van group: cultured with vancomycin (Solarbio, China), (1g per person ${ }^{11,12}=14.28 \mu \mathrm{g} /$ $\mathrm{mL}$ ), Tob group: cultured with tobramycin (Solarbio, China), $\left(2 \mathrm{~g}\right.$ per person $\left.{ }^{3,13}=28.57 \mu \mathrm{g} / \mathrm{mL}\right), \mathrm{Van}+$ Tob group (vancomycin $14.28 \mu \mathrm{g} / \mathrm{mL}$ combined with tobramycin $28.57 \mu \mathrm{g} / \mathrm{mL})$.

\section{LIVE/DEAD Assay}

The LIVE/DEAD kit (L3224, InvitrogenTM, USA) was used to evaluate the viability of BMSCs. The cells were cultured in the media with or without antibiotics for about 7 days and then incubated with calcein AM and ethidium homodimer solution for 30mins. The cells were then imaged under fluorescence microscopy.

\section{Cell Proliferation Assay}

The proliferation rates of cells cultured with different antibiotics were detected by CCK-8 assay (Dojindo, Tokyo, Japan) after BMSCs were cultured in different antibiotics media for $1,3,5$, or 7 days. BMSCs cultured without any antibiotic served as the control. The absorbance at $450 \mathrm{~nm}$ was read with a microplate reader.

\section{Alizarin Red S (ARS) Staining}

BMSCs were cultured in osteogenic media with or without antibiotics for 14 and 21 days, the cells were fixed in ethanol and then stained with $40 \mathrm{mM}$ Alizarin Red S (Sigma-Aldrich, USA), $\mathrm{pH} 4.0$, for $20 \mathrm{~min}$. Cells were rinsed with doubledistilled $\mathrm{H} 2 \mathrm{O}$ (ddH2O). Then, 10\% acetic acid (SigmaAldrich, USA) was used to elute the stain, followed by $10 \%$ ammonium hydroxide (Sigma-Aldrich, USA). Finally, the optical densities were read at each time point (14 and 21 days).

\section{Alkaline Phosphatase (ALP) Staining}

The expression of ALP at 14 and 21 days was assessed using an ALP kit (Yeasen, China). After osteogenic induction, the BMSCs were fixed with ice-cold methanol for 30 mins. The fixed BMSCs were incubated in the BCIP/NBT solution and then observed under a light microscope.

\section{Wound Healing Assay}

The cell migration ability was assessed by wound healing assay. Monolayer cells were seeded in a 6-well plate and scratched a line in the same area of each well by a pipet tip. The suspended cells were removed gently by PBS. Then, the serum-free medium was added into the plates and incubated for $24 \mathrm{hrs}$. Differences in the healed scratch wound area were compared to assess cell migration by using Image $\mathrm{J}$. 


\section{Quantitative Real-Time (qRT)-PCR}

qRT-PCR was performed to evaluate the osteogenic mRNA (Col-1, Runx2, ALP, OCN, OPN, and SOST) expression levels in the BMSCs after 14 and 21 days of Osteogenic induction. The total mRNA was extracted and transcribed into cDNA using a cDNA Synthesis Kit (Thermo-Fisher). The primers used are listed in Table 1. The expression levels of osteoblastic genes were calculated based on the $2-\Delta \Delta \mathrm{Ct}$ method.

\section{Statistical Analysis}

All statistical analyses were performed by Prism software 8.2 (GraphPad) for macOS. Data were shown as mean \pm standard deviation (SD). One-way analysis of variance and Student's $t$-test were used to compare the differences. All the results were biologically replicated three times. All reported $p$ values are 2 -sided, and values of $p<0.05$ were considered as statistically significant.

\section{Results}

\section{Analysis of BMSCs Viability and Proliferation}

After culturing for 7 days, BMSCs viability was assessed by Live/Dead imaging. As shown in Figure 1A-D. The red and green fluorescence are presented as dead and live cells, respectively. A slight increase number of dead cells (red) were found in the Van and Van+Tob group. However, there were no statistically significant (Figure $1 \mathrm{E}, p=0.455, p=0.382$ ). The viability of BMSCs in four groups was all above $92 \%$. A CCK8 assay was used to investigate the proliferation rate of BMSCs over 7 days (Figure 1F). The cell number steadily increased up to 7 days in the four groups and no significant differences were found among four groups at each time point. These results indicate that the viability and proliferation of BMSCs were not affected by vancomycin and/or tobramycin.

\section{Antibiotics Affect Osteogenic Differentiation of BMSCs ARS and ALP Staining}

After osteogenic induction for 14 and 21 days, ARS and ALP staining were performed to detect the calcium deposition and ALP activity, respectively. The ARS staining showed that calcium deposition was enhanced under tobramycin, but reduced significantly under vancomycin at both 14 and 21 days (Figure 2A). The ALP staining showed that the Van group was lower than the other three groups, and the Tob group was higher than the others (Figure 2C). However, quantitative analysis found that both the ARS and ALP staining in the Van+Tob group were higher than the Van group (Figure 2B and D). These results indicated that vancomycin inhibited calcium deposition of BMSCs, which could rescue by tobramycin.

\section{Antibiotics Affect BMSCs Migration}

Wound healing assay was performed to explore the effects of vancomycin and tobramycin on the migration of BMSCs. The results showed that the vancomycin significantly inhibited the motility of BMSCs. However, the area of healed scratch wound in the Tob group was bigger than the other three groups; and the area of healed scratch wound in the Van +Tob group was bigger than the Van group (Figure 3). These results indicated that tobramycin could rescue reduced cell migration of BMSCs caused by vancomycin.

\section{Antibiotics Affect the Osteogenic mRNA Expression of BMSCs}

The osteogenesis-related mRNA expression levels of Col-1, ALP, Runx2, OCN, OPN, SOST were determined by qRTPCR assay at both 14 and 21 days. As shown in Figure 4, all the osteogenesis-related mRNA increased from 14 to 21 days. On day 14, the Col-1, Runx2, ALP, OCN in the Tob

Table I Primer Pair Sequences for qRT-PCR

\begin{tabular}{|l|l|l|}
\hline \multirow{2}{*}{ Gene } & Sequences $\left(\mathbf{5}^{\prime} \mathbf{- 3}^{\prime} \mathbf{\prime}\right)$ & Reverse \\
\cline { 2 - 3 } & Forward & TCCACCACCCTGTTGCTGTA \\
\hline GAPDH & GACTTCAACAGCAACTCCCAC & GTCAAGGCTGAGAACGGGAA \\
Runx2 & CCTCGCTTTCCTTCCTCTCC & CAGGTACGTGTGGTAGTGAGT \\
ALP & TTCTCCAACCCACGAATGCAC & GGAAACGCAGGATTTCCCAC \\
OCN & TCCTGTTGACACCCCAAACC & CGCCTGGGTCTCTTCACTAC \\
OPN & CTCACACTCCTCGCCCTATTG & CACACTATCACCTCGGCCATC \\
SOST & ATCTCCTAGCCCCACAGACCC & GGCCCATCGGTCACGTAG \\
\hline
\end{tabular}



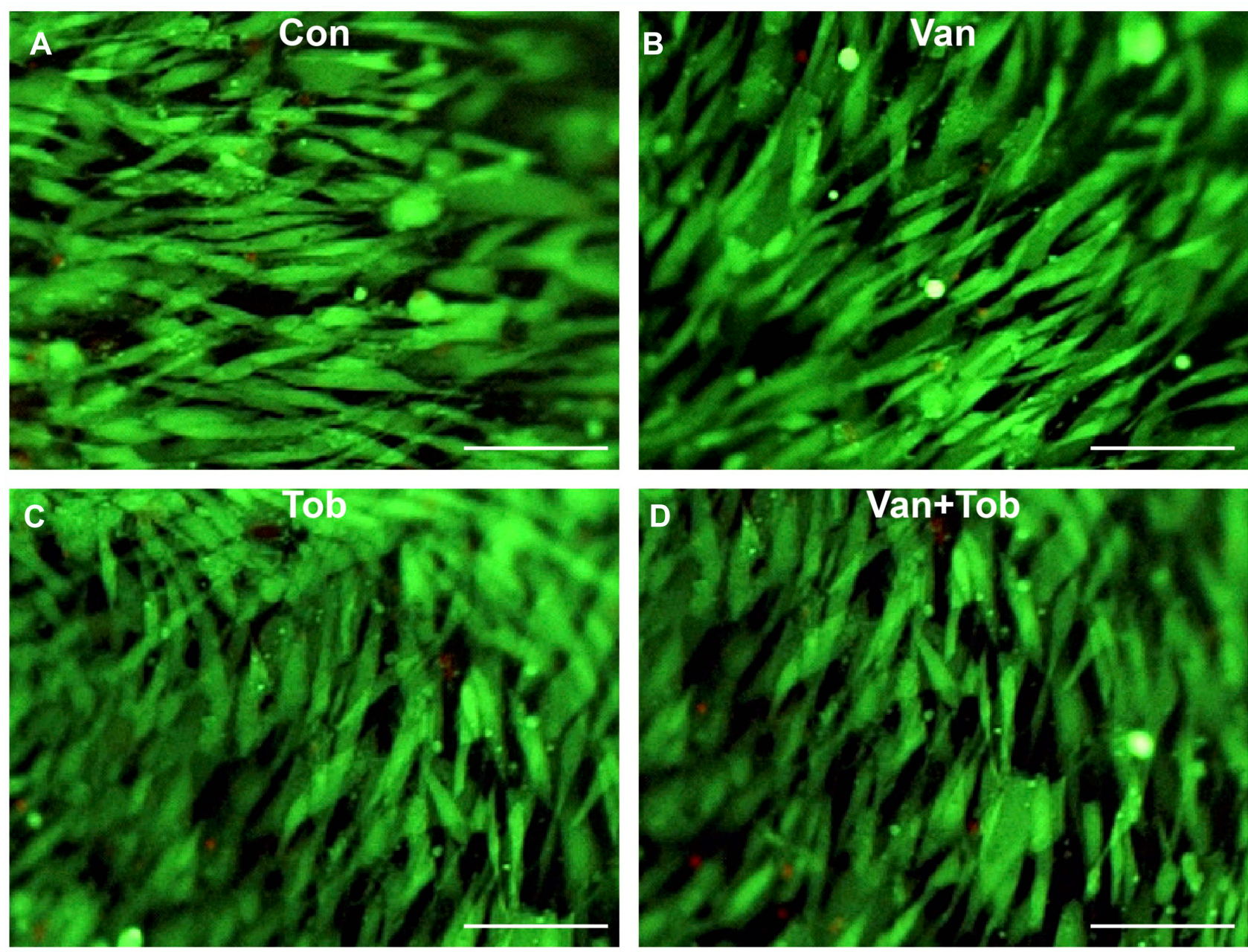

E

Live-Dead Cells

$\mathbf{F}$

CCK8
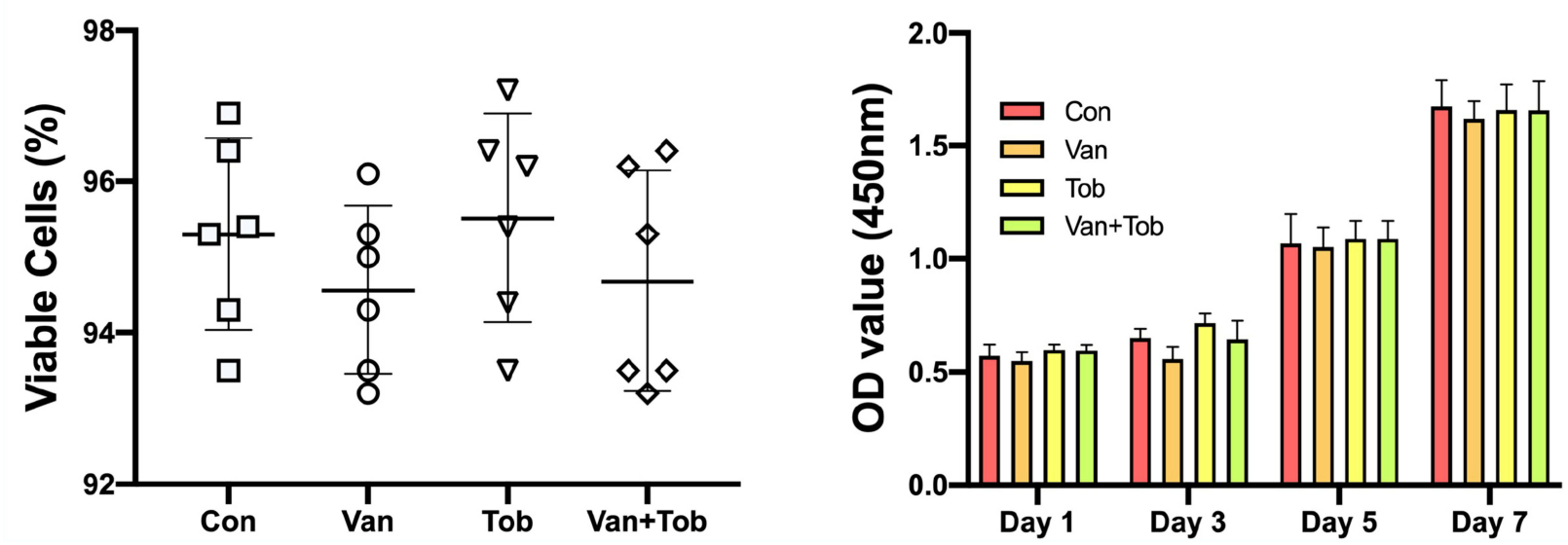

Figure I Cell viability and proliferation. The Live/Dead staining of BMSCs cultured without antibiotics on the 7 days (A); The Live/Dead staining of BMSCs cultured with vancomycin (B), tobramycin (C), and combination of vancomycin-tobramycin (D) on the 7 days (scale bars, 50 $\mu \mathrm{m}$ ). The quantitative results of the Live/Dead assay (E). Quantitative analysis of BMSCs proliferation rate by CCK-8 assay over 7 days (F).

Abbreviations: Con, control; Van, vancomycin; Tob, tobramycin.

group were higher than the Con group. And Runx2, ALP, and OCN in the Van+Tob group were higher than the Van group. At 21 days, all markers were highest in the Tob group, but lowest in the Van group. Compared with the Van group, the Van+Tob group showed markedly higher mRNA expression at day 21 . These results implied that 

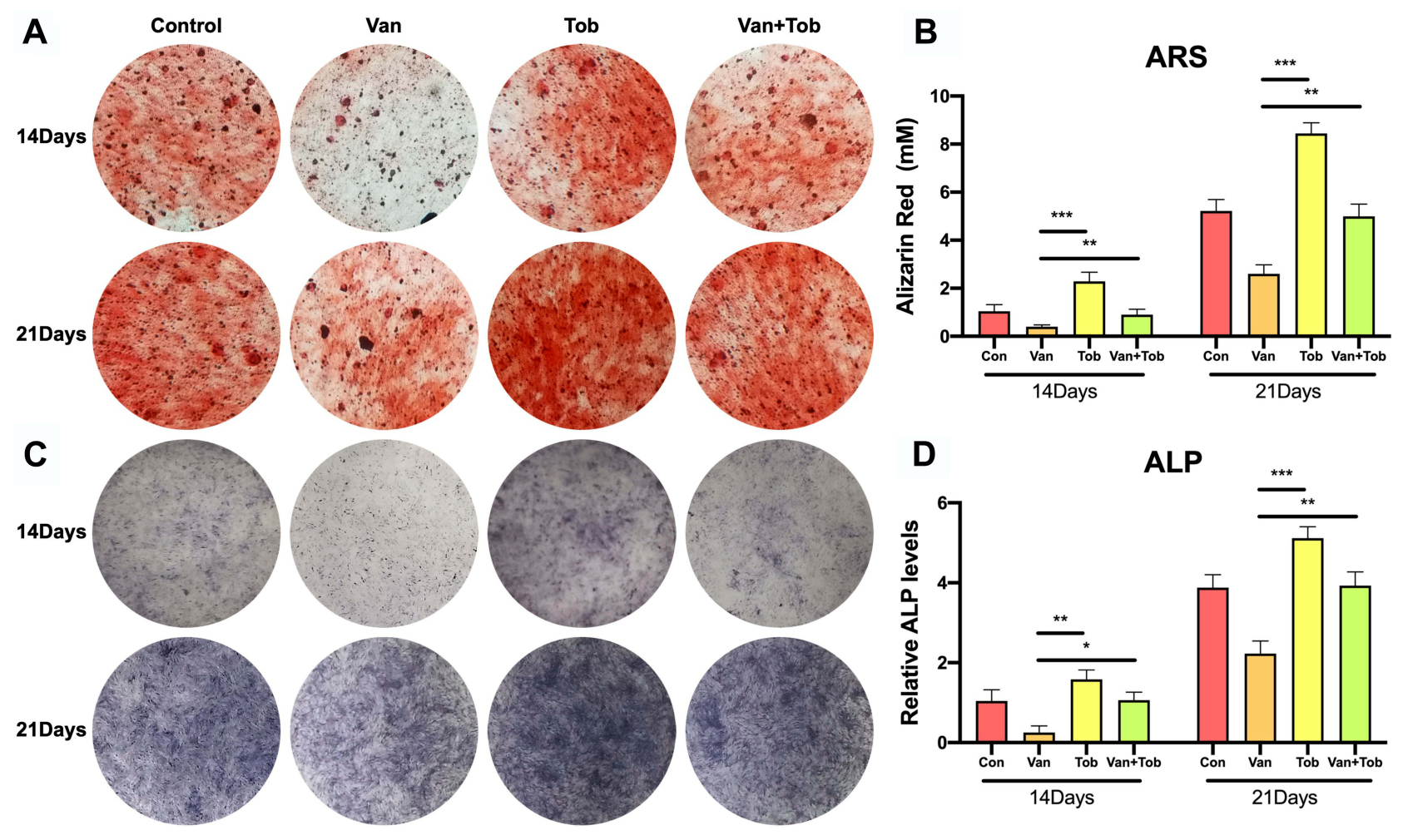

Figure 2 The effect of vancomycin and tobramycin on mineralization of BMSCs was assessed by ARS and ALP staining. BMSCs were stained with ARS and ALP after cultured 14 and 21 days, respectively. ARS staining (A) and its quantification (B) in the Con, Van, Tob, and Van+Tob groups. ALP staining (C) and its quantitative analysis (D) in the four groups. ${ }^{*} p<0.05, *^{*} p<0.01$, *** ${ }^{*}<0.001$.

Abbreviations: Con, control; Van, vancomycin; Tob, tobramycin.

vancomycin inhibited the osteogenesis of BMSCs, while the tobramycin offsets the osteogenic ability of BMSCs which reduced by vancomycin.

\section{Discussion}

Clinically, local delivery of vancomycin combined with tobramycin was wildly used for open fracture and bone tissue infection. ${ }^{14,16}$ This dual antibiotic therapy is an effective and safe method to reduce bacterial infections at a traumatic wound and may reduce the undesirable effects related to the systemic administration and the risk of resistance. Additionally, a study has proved that local delivery of vancomycin and tobramycin have less cytotoxic and did not appreciably affect cell number. ${ }^{17}$ As expected, the results of CCK8 and Live/Dead assay shown that local administration of vancomycin, tobramycin, or vancomycin combined with tobramycin did not affect cell viability and proliferation of BMSCs in our study. Furthermore, we have demonstrated that local delivery of vancomycin may have detrimental effects on osteogenesis of BMSCs. However, tobramycin may promote bone regeneration in vitro. Additionally, results also shown that the combination of vancomycin-tobramycin had no inhibitory effect on osteogenesis.

Eder et.al ${ }^{9}$ have proved that local application of vancomycin may reduce bone regeneration in vitro. They cultured vancomycin with osteoblast cells and found that the migration of osteoblast, synthesis of ALP, calcium deposition was significantly reduced by vancomycin. Our results also showed that ARS staining, ALP staining, migration of BMSCs, as well as the osteogenesis-related genes were reduced in Van group. Ishida et $\mathrm{al}^{3}$ investigated the effects of vancomycin on osteogenesis in a rat model and found that vancomycin affects bone fusion. In contrast, another in vivo study concluded that vancomycin combined with recombinant human bone morphogenetic protein-2 (rhBMP-2) did not affect bone fusion. ${ }^{12}$ The rhBMP-2 may offset the negative impact of vancomycin on bone regeneration. But the application of rhBMP-2 may result in heterotopic ossification in some orthopedic surgeries. ${ }^{18}$ Our in vitro experiments confirmed that vancomycin reduced the osteogenesis process.

Our findings also proved that the tobramycin has better bone regeneration effect than the control group in vitro. Recently, Rao et al ${ }^{19}$ found that tobramycin could promote 

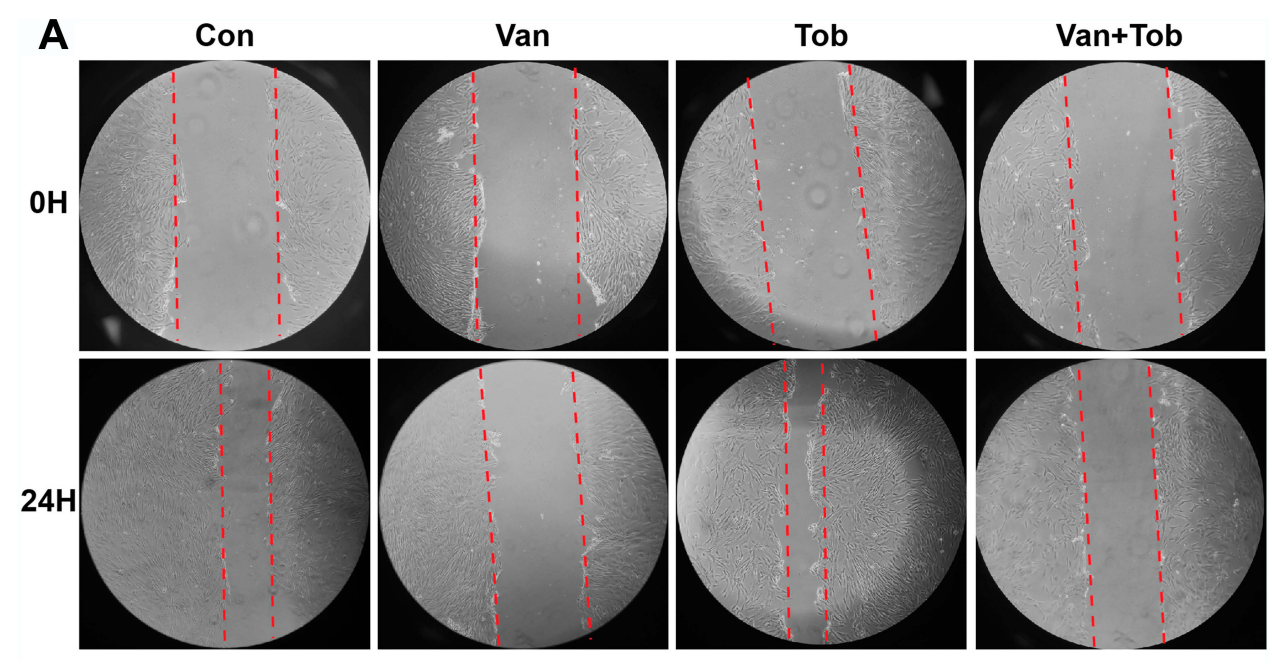

B

Scratch

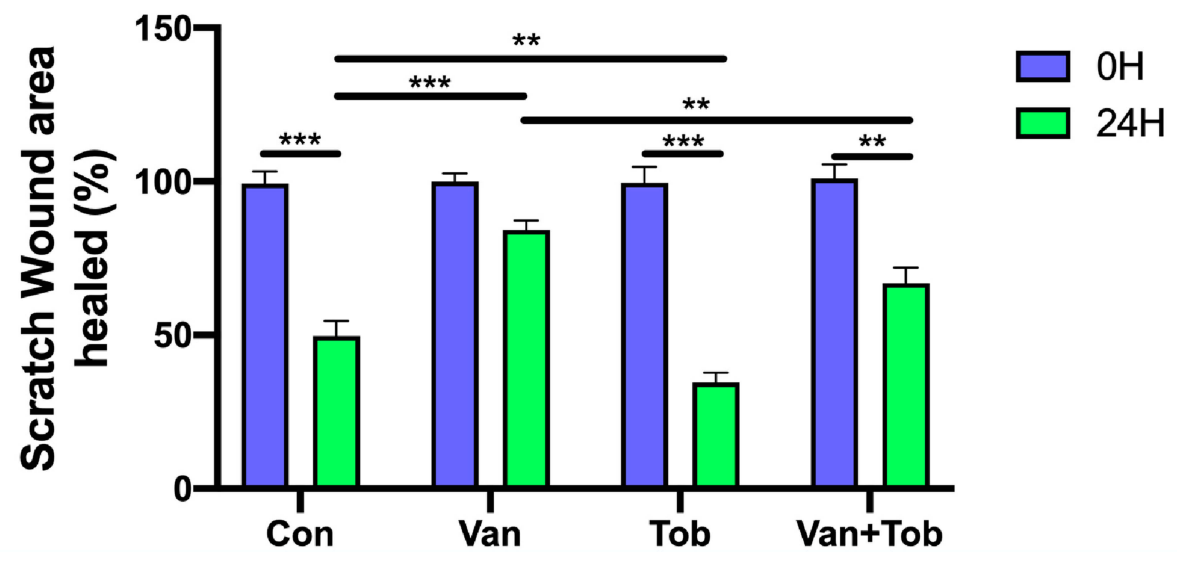

Figure 3 Antibiotics affect the migration of BMSCs. In vitro scratch assays were used to analyzed the migration rate of BMSCs with different antibiotics within 24 hours (A). Statistical analysis of scratch assay (B). **p $<0.01$, **** $<0.001$.

Abbreviations: Con, control; Van, vancomycin; Tob, tobramycin.

fracture healing by accelerating osteogenesis differentiation of MSCs through the Wnt/ $\beta$-catenin pathway. Another in vivo study also found that utilizing tobramycin powder might be beneficial for bone fusion. ${ }^{3}$ A comparative study of tobramycin and vancomycin impregnated material implanted into a rabbit tibia model. The results showed that tobramycin has better osteogenesis scores than vancomycin. All of these studies are comparable with our findings. Tobramycin is beneficial for patients who are prone to Gram-negative bacteria infection. However, for the MRSA bacterial infection, vancomycin powder is more useful. Because the presence of tobramycin has a synergistic-like effect on bactericidal activity of vancomycin, ${ }^{13}$ several studies recommended local delivery of the combination of vancomycin-tobramycin powder to prevent infection. ${ }^{6,20,21}$ In the current study, we investigated the osteogenesis effect of a combination of vancomycin-tobramycin. The in vitro experiments showed that the BMSCs in the Van+Tob group have almost the same osteogenic ability as the control group, which are better than Van group. These results indicated that the tobramycin could counteract the negative effect of vancomycin on osteogenesis. Thus, the combined application of vancomycin and tobramycin powder could cover the majority of pathogens and reduce infection and nonunion rate in orthopedic surgery.

There are several limitations in this study. First, only two antibiotics were included in our study. The osteogenesis of other antibiotics, such as gentamicin, ${ }^{22}$ ampicillin, ${ }^{23}$ and amikacin, ${ }^{24}$ should be further explored. Second, we only use the clinical concentration of two antibiotics in the current study. Additional experiments of multiple concentrations should be studied, which allow us to find the proper ratio of vancomycin to tobramycin and recommended doses for patients. Third, since the concentration of antibiotics is 
A

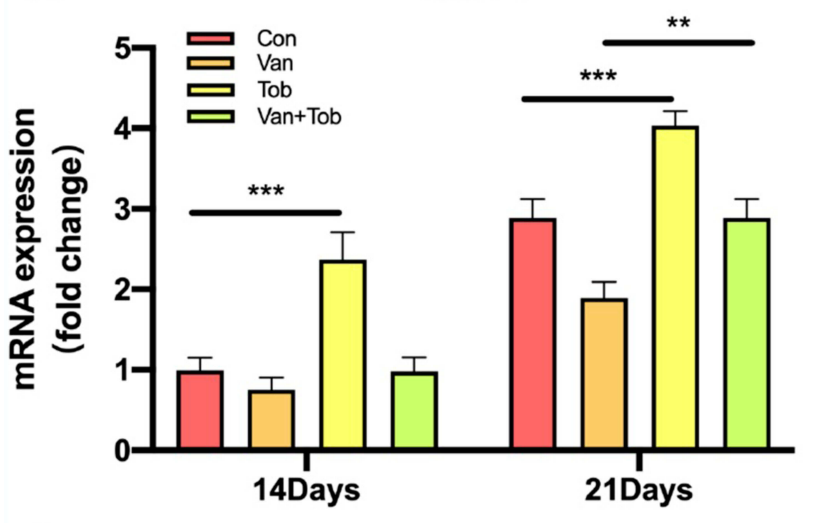

C

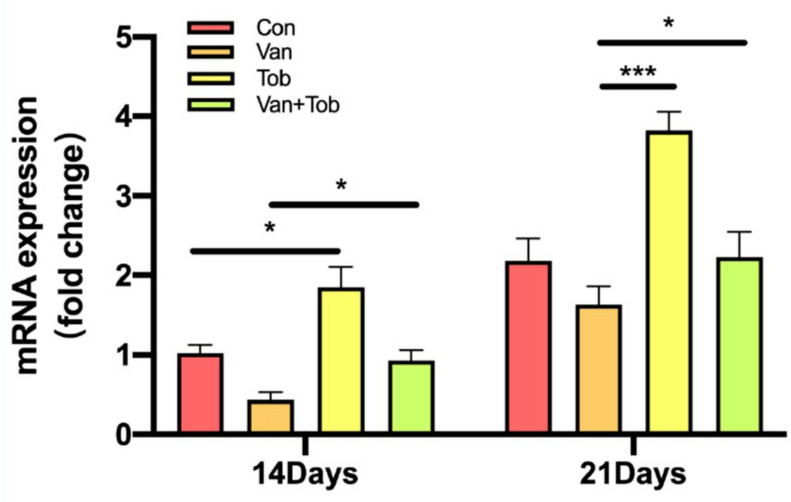

E

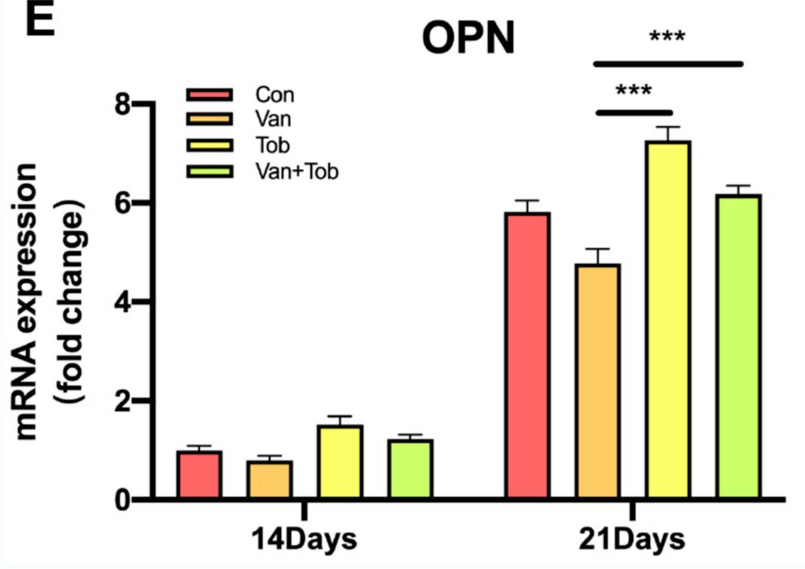

B

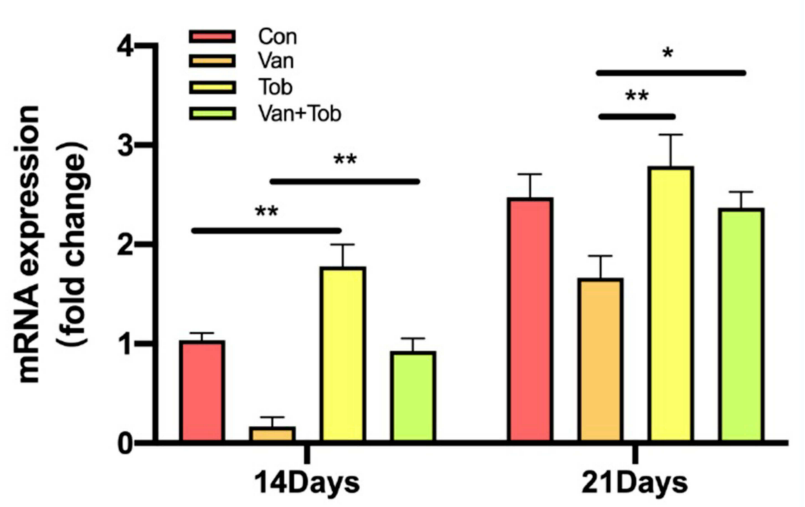

D

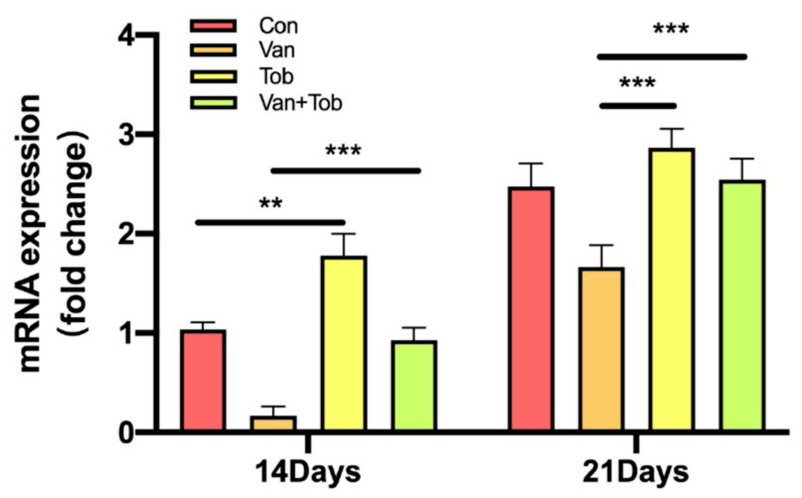

$\mathbf{F}$

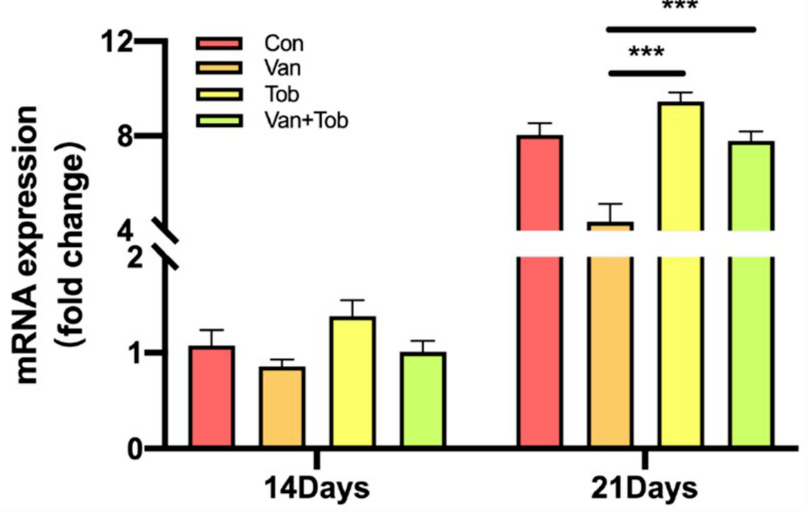

Figure 4 Real-time PCR assay was applied to evaluate the expression of osteogenic mRNA. The osteogenic mRNA expression of Col-I (A), Runx2 (B), ALP (C), OCN (D), OPN (E), SOST (F) in BMSCs was quantified by real-time PCR after 14 and 21 days of incubation. ${ }^{*} p<0.05$, **p $<0.01$, *** $p<0.001$.

Abbreviations: Con, control; Van, vancomycin; Tob, tobramycin.

different when it is used in vivo, preclinical experiments should be performed in the near further. Furthermore, our results proved the osteogenesis of the local delivery of antibiotics. But the bacterial infection was not addressed in this study. We would like to investigate these in our future studies.

In conclusion, local delivery of vancomycin might reduce the bone regeneration ability of BMSCs, while vancomycin combined with tobramycin does not affect the osteogenic potential of BMSCs. These results suggested that appropriate randomized blinded prospective clinical trials are needed to exam the safety and efficacy of the combination of vancomycin-tobramycin in reducing the risk of both nonunion and surgical site infections in orthopedic surgery. 


\section{Funding}

This research was funded by Zhongwen Wang Academic Experience Heritage Studio Special Fund of Guangdong Provincial Hospital of Traditional Chinese Medicine (2018. No.7); Guangdong Provincial Famous Doctor of Traditional Chinese Medicine Heritage Studio Special Fund of Guangdong Provincial Hospital of Traditional Chinese Medicine (The Second Affiliated Hospital of Guangzhou University of Chinese Medicine) (2017. No.17) and Initial Scientific Project of Beijing Friendship Hospital (yyqdkt2019-9).

\section{Disclosure}

The authors report no conflicts of interest in this work.

\section{References}

1. Sweet FA, Forsthoefel CW, Sweet AR, Dahlberg RK. Local versus systemic antibiotics for surgical infection prophylaxis in a rat model. $J$ Bone Joint Surg Am. 2018;100(18):e120. doi:10.2106/JBJS.18.00105

2. Pais GM, Liu J, Avedissian SN, et al. Scheetz. Lack of synergistic nephrotoxicity between vancomycin and piperacillin/tazobactam in a rat model and a confirmatory cellular model. J Antimicrob Chemother. 2020;75(5):1228-1236. doi:10.1093/jac/dkz563

3. Ishida W, Perdomo-Pantoja A, Elder BD, et al. Effects of intraoperative intrawound antibiotic administration on spinal fusion: a comparison of vancomycin and tobramycin in a rat model. J Bone Joint Surg Am. 2019;101(19):1741-1749. doi:10.2106/JBJS.18.00988

4. Edelstein AI, Weiner JA, Cook RW, et al. Intra-articular vancomycin powder eliminates methicillin-resistant $\mathrm{S}$. aureus in a rat model of a contaminated intra-articular implant. J Bone Joint Surg Am. 2017;99 (3):232-238. doi:10.2106/JBJS.16.00127

5. Haimoto S, Schar RT, Nishimura Y, Hara M, Wakabayashi T, Ginsberg HJ. Reduction in surgical site infection with suprafascial intrawound application of vancomycin powder in instrumented posterior spinal fusion: a retrospective case-control study. $J$ Neurosurg Spine. 2018;29(2):193-198. doi:10.3171/2017.12.SPINE17997

6. Slane J, Gietman B, Squire M. Antibiotic elution from acrylic bone cement loaded with high doses of tobramycin and vancomycin. $J$ Orthop Res. 2018;36:1078-1085.

7. Aljutayli A, Marsot A, Nekka F. An Update on Population Pharmacokinetic Analyses of Vancomycin, Part I: in Adults. Clin Pharmacokinet. 2020;59(6):671-698. doi:10.1007/s40262-02000866-2

8. Palser S, Smith S, Nash EF, Agarwal A, Smyth AR, Buchbinder R. Treatments for preventing recurrence of infection with Pseudomonas aeruginosa in people with cystic fibrosis. Cochrane Database Syst Rev. 2019;12:CD012300. doi:10.1002/14651858.CD013502

9. Eder C, Schenk S, Trifinopoulos J, et al. Does intrawound application of vancomycin influence bone healing in spinal surgery? Eur Spine $J$. 2016;25(4):1021-1028. doi:10.1007/s00586-015-3943-9
10. Seavey JG, Wheatley BM, Pavey GJ, et al. Early local delivery of vancomycin suppresses ectopic bone formation in a rat model of trauma-induced heterotopic ossification. J Orthop Res. 2017;35 (11):2397-2406. doi:10.1002/jor.23544

11. Adogwa O, Elsamadicy AA, Sergesketter A, et al. Prophylactic use of intraoperative vancomycin powder and postoperative infection: an analysis of microbiological patterns in 1200 consecutive surgical cases. J Neurosurg Spine. 2017;27(3):328-334. doi:10.3171/2017.2. SPINE161310

12. Mendoza MC, Sonn KA, Kannan AS, et al. The effect of vancomycin powder on bone healing in a rat spinal rhBMP-2 model. J Neurosurg Spine. 2016;25(2):147-153. doi:10.3171/2015.11.SPINE15536

13. González Della A, Valle M, Bostrom B, Brause C, Salvati EA. Salvati. Effective bactericidal activity of tobramycin and vancomycin eluted from acrylic bone cement. Acta Orthop Scand. 2001;72 (3):237-240. doi:10.1080/00016470152846547

14. Shekhar C. An innovative technique in local antibiotic delivery method in open infected wounds of the musculoskeletal system. Int $J$ Low Extrem Wounds. 2019;18(2):153-160. doi:10.1177/1534 734619841764

15. Owen MT, Keener EM, Hyde ZB, et al. Intraoperative topical antibiotics for infection prophylaxis in pelvic and acetabular surgery. $J$ Orthop Trauma. 2017;31(11):589-594. doi:10.1097/BOT.000000000 0000941

16. Tennent DJ, Shiels SM, Jennings JA, Haggard WO, Wenke JC. Local control of polymicrobial infections via a dual antibiotic delivery system. J Orthop Surg Res. 2018;13(1):53. doi:10.1186/s13018-018-0760-y

17. Rathbone CR, Cross JD, Brown KV, Murray CK, Wenke JC. Effect of various concentrations of antibiotics on osteogenic cell viability and activity. J Orthop Res. 2011;29(7):1070-1074. doi:10.1002/ jor.21343

18. de Guzman RC, Saul JM, Ellenburg MD, et al. Bone regeneration with BMP-2 delivered from keratose scaffolds. Biomaterials. 2013;34(6):1644-1656. doi:10.1016/j.biomaterials.2012.11.002

19. Rao M, Pang X, Yang J. Tobramycin promotes fracture healing by accelerating osteogenesis differentiation of MSCs through activating Wnt/beta-catenin pathway. Minerva Med. 2019;111:189.

20. Nart J, de Tapia B, Pujol A, Pascual A, Valles C. Vancomycin and tobramycin impregnated mineralized allograft for the surgical regenerative treatment of peri-implantitis: a 1-year follow-up case series. Clin Oral Investig. 2018;22(6):2199-2207. doi:10.1007/s00784-0172310-0

21. Sanicola SM, Albert SF. The in vitro elution characteristics of vancomycin and tobramycin from calcium sulfate beads. J Foot Ankle Surg. 2005;44(2):121-124. doi:10.1053/j.jfas.2005.01.006

22. Anagnostakos K, Meyer C. Antibiotic elution from hip and knee acrylic bone cement spacers: a systematic review. Biomed Res Int. 2017;2017:4657874.

23. Takeuchi M, Wakao N, Kamiya M, Hirasawa A, Murotani K, Takayasu M. A double-blind randomized controlled trial of the local application of vancomycin versus ampicillin powder into the operative field for thoracic and/or lumbar fusions. J Neurosurg Spine. 2018;29(5):553-559. doi:10.3171/2018.3.SPINE171111

24. Boles LR, Awais R, Beenken KE, Smeltzer MS, Haggard WO, Jessica AJ. Local delivery of amikacin and vancomycin from chitosan sponges prevent polymicrobial implant-associated biofilm. Mil Med. 2018;183(suppl_1):459-465. doi:10.1093/milmed/usx161 


\section{Publish your work in this journal}

Infection and Drug Resistance is an international, peer-reviewed openaccess journal that focuses on the optimal treatment of infection (bacterial, fungal and viral) and the development and institution of preventive strategies to minimize the development and spread of resistance. The journal is specifically concerned with the epidemiology of antibiotic resistance and the mechanisms of resistance development and diffusion in both hospitals and the community. The manuscript management system is completely online and includes a very quick and fair peerreview system, which is all easy to use. Visit http://www.dovepress.com/ testimonials.php to read real quotes from published authors. 